Pacific Journal of Mathematics

NON-CONTINUOUS DEPENDENCE OF SURFACES OF LEAST 


\title{
NON-CONTINUOUS DEPENDENCE OF SURFACES OF LEAST AREA ON THE BOUNDARY CURVE
}

\author{
Michael BeEson
}

\begin{abstract}
In this paper, we consider the question of continuous dependence associated with the following version of Plateau's problem: Given a (sufficiently smooth) Jordan curve $\Gamma$, find a surface of least area bounded by $\Gamma$. In other words, we ask whether a surface $S_{\Gamma}$ of least area among surfaces bounded by $\Gamma$ can be found, continuously in $\Gamma$. The answer is no; in fact, sometimes one does not even have local continuous dependence. That is, for certain curves $\Gamma_{0}$, one cannot find $S_{\Gamma}$ continuously in $\Gamma$, even on any neighborhood of $\Gamma_{0}$.
\end{abstract}

We became interested in this question through our interest in constructive aspects of Plateau's problem. A general principle is that if a problem can be constructively solved, then the solution depends (at least locally) continuously on the parameters provided the parameters come from a complete metric space). This may be seen intuitively as follows: if a computer is to compute the solution to accuracy $\epsilon$, it must be able to do so on the basis of an approximate value of the parameters. This is closely related to Hadamard's formulation of the concept of a "well-posed problem". In [1] we have made a metamathematical study of this "Principle of Local Continuity". It follows from the results there, together with the main theorem of this paper, that no proof of Plateau's problem as stated above (say for $C^{(n)}$ boundaries) can be given in known constructive formal systems; see [2] for details.

There is a weaker form of Plateau's problem, in which it is required to find, not a surface of least area, but a surface which is a critical point of the area functional; that is to say, a minimal surface. It was proved by Hildebrant and again by Tomi [8] that for analytic boundary curves, this problem can be locally continuously solved. This lends evidence to support the conjecture that this version of Plateau's problem can be constructively solved, at least for sufficiently smooth boundaries. It is almost certainly true that one does not have global continuous dependence of a minimal surface on the boundary curve, even though one does have local continuous dependence, but this has yet to be proved."

For the sake of precision, we state exactly what is meant by "local

Added in proof: This follows from the results of A. J. Tromba and the author, the cusp catastrophe of Thom in the bifurcation of minimal surfaces, to appear. 
continuous dependence'. Consider the problem of finding an $x$ such that $P(a, x)$. Here $a$ are the parameters of the problem; for example, $a$ might be a boundary curve and $x$ a surface of least area bounded by $a$. Let $N_{\delta}(a)$ denote the neighborhood of $a$ of radius $\delta$. We say the problem can be solved locally continuously if

$$
\forall a \exists x \forall \epsilon>0 \exists \delta>0 \forall b \in N_{\delta}(a) \exists y \in N_{\epsilon}(x)(P(a, x) \& P(b, y))
$$

That is, $x$ is a solution stable under small perturbations $b$ of the parameter $a$. It is worth remarking that this is not the strongest possible notion - one could ask for a neighborhood $N(a)$ and a continuous functional defined on that neighborhood and producing a solution of the problem. Since we are proving a negative result in this paper, we take the weaker formulation. This is also the formulation taken in Tomi's paper [8], where a positive result is proved. The proof there does not yield a continuous functional on a neighborhood of a given boundary curve.

We now proceed to a motivation and description of our proof. We begin with some remarks on the analogy between minimizing the area functional and minimizing some differentiable function $f$ from $[0,1]$ to the reals. Such an $f$ has a minimum value on $[0,1]$, but we cannot in general compute explicitly an $x$ such that $f(x)$ is a minimum. The reason is, that $f$ may have two relative minima, say at $x=1 / 3$ and $x=2 / 3$, and on the basis of finer and finer approximations to $f$, we can't decide which of the two is an absolute minimum. That is, the value $x$ at which $f$ takes its minimum value does not depend continuously on $f$; let $f_{0}$ have two relative minima, as above, but $f_{0}(1 / 3)=f_{0}(2 / 3)$; then in any neighborhood of $f_{0}$, there will be some functions whose only absolute minimum is at $1 / 3$, and some whose only absolute minimum is at $2 / 3$. We shall show that the situation with the area functional is similar.

The example suggests that we should look for a contour $\Gamma=\Gamma_{t}$ (depending on a real parameter $t$ ) such that the area functional has two relative minima among surfaces bounded by $\Gamma$, and which one of the two is an absolute minimum depends on the sign of $t$. Thus, we are led to examine curves $\Gamma$ bounding more than one minimal surface. Several examples are commonly used to demonstrate the non-uniqueness of solutions to Plateau's problem. Three of these are illustrated in Fig. 1. Note that each curve in Fig. 1 is preserved under the symmetry $\sigma$ which rotates by $90^{\circ}$ around the $z$ axis and then reflects in the $x-y$ plane, as well as under $\rho_{x}$ and $\rho_{y}$ (reflection in the $y-z$ plane and $x-z$ plane, respectively). Soap film experiments with wires bent in the shapes of these contours suggest that each of these curves bounds exactly three minimal surfaces (of the type of the disk); two relative minima and one unstable surface. For instance, if $\Gamma_{0}$ is the curve in Figure 1a, one 
relative minimum, say $S$, goes from the upper left, down around the bottom, and back up on the right; the other, say $R$, is its image under the symmetry $\sigma$ : it starts in the back bottom, comes up over the top, and down in the front. The unstable surface bounded by $\Gamma_{0}$ is a segment of Enneper's surface, with a saddle point at the origin. Now, if we define perturbations $\Gamma_{t}$ of $\Gamma_{0}$ so that for $t>0$, the top parts of $\Gamma_{t}$ are pinched together, and for $t<0$, the top parts are pulled apart, then presumably there are surfaces $R_{t}$ and $S_{t}$, such that as $t$ changes sign, the absolute minimum of area jumps from $R_{t}$ to $S_{t}$.

There are a number of difficulties with this plan. First, and most important, it is not known that $\Gamma$ bounds only these three minimal surfaces. In each of the cases illustrated in Figure 1, it is known that $\Gamma$ bounds at least three minimal surfaces, but (except in cases of uniqueness) not a single boundary curve is known for which an explicit estimate of the number of minimal surfaces with that boundary can be given. (If $\Gamma$ is analytic, the number of relative minima bounded by $\Gamma$ is known to be finite, but nobody knows how to compute even this number.) Even if

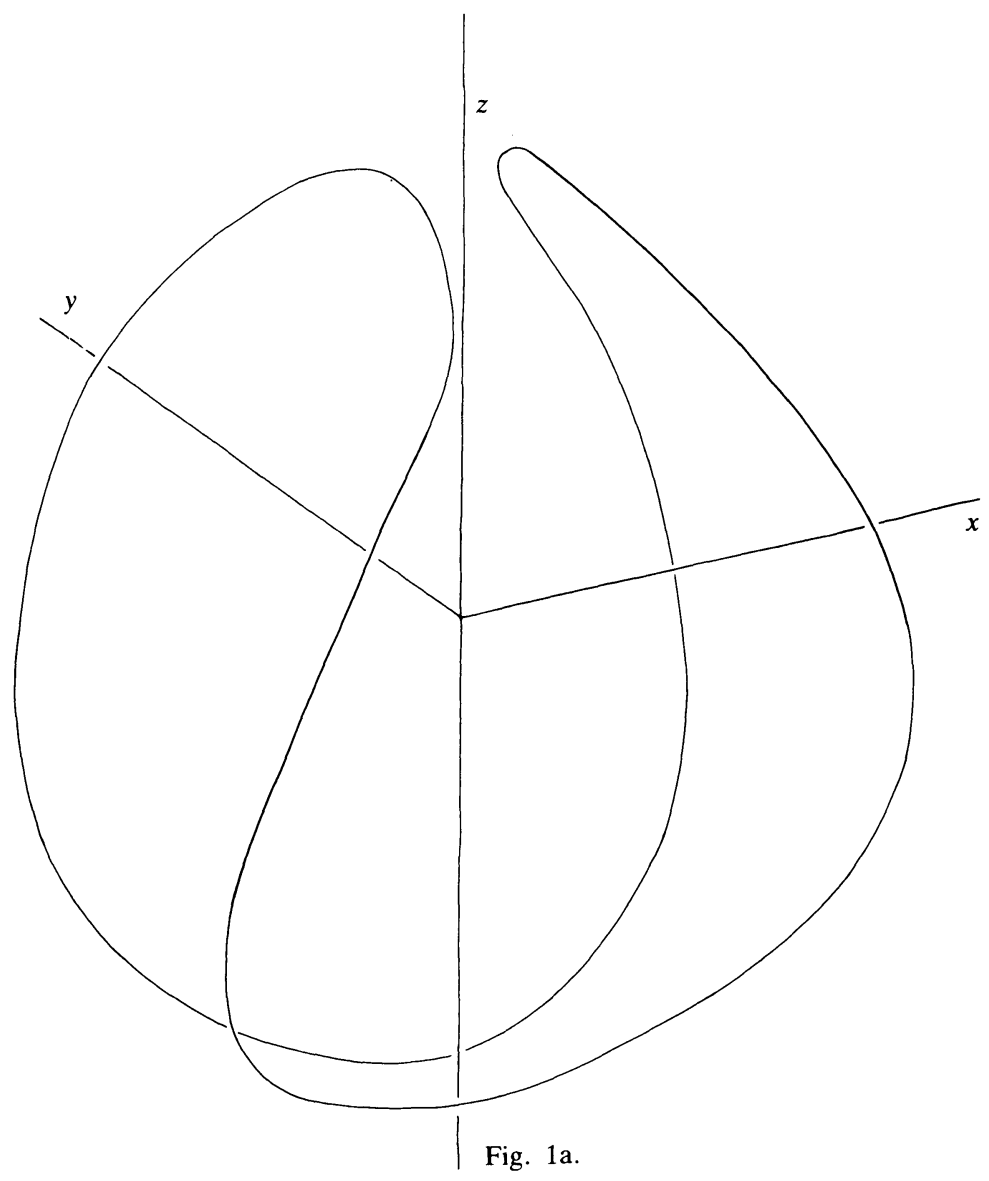




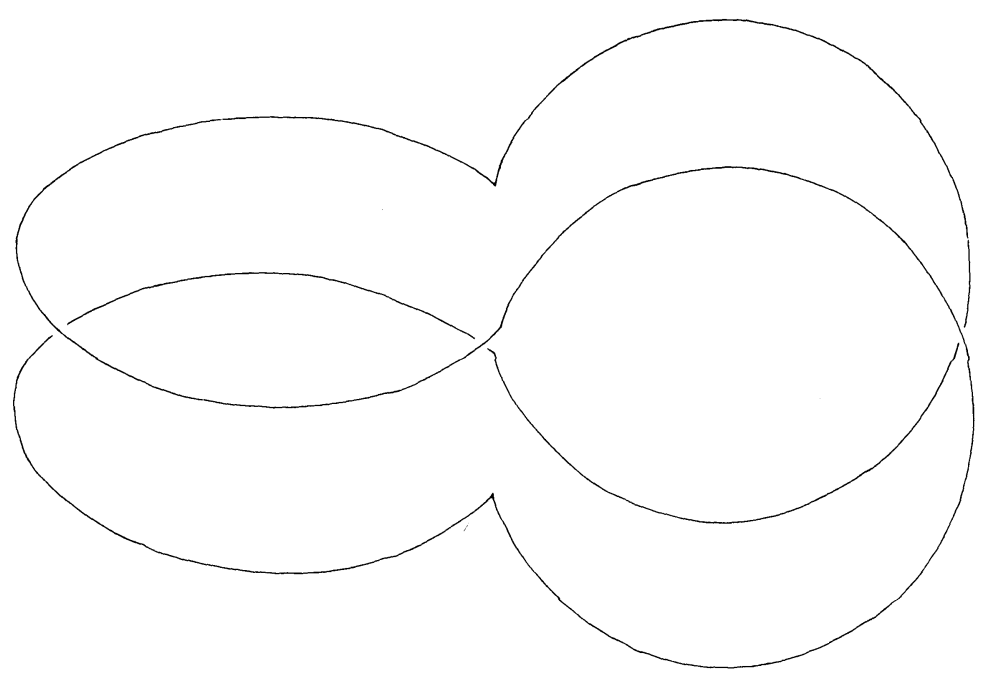

Fig. $1 b$.

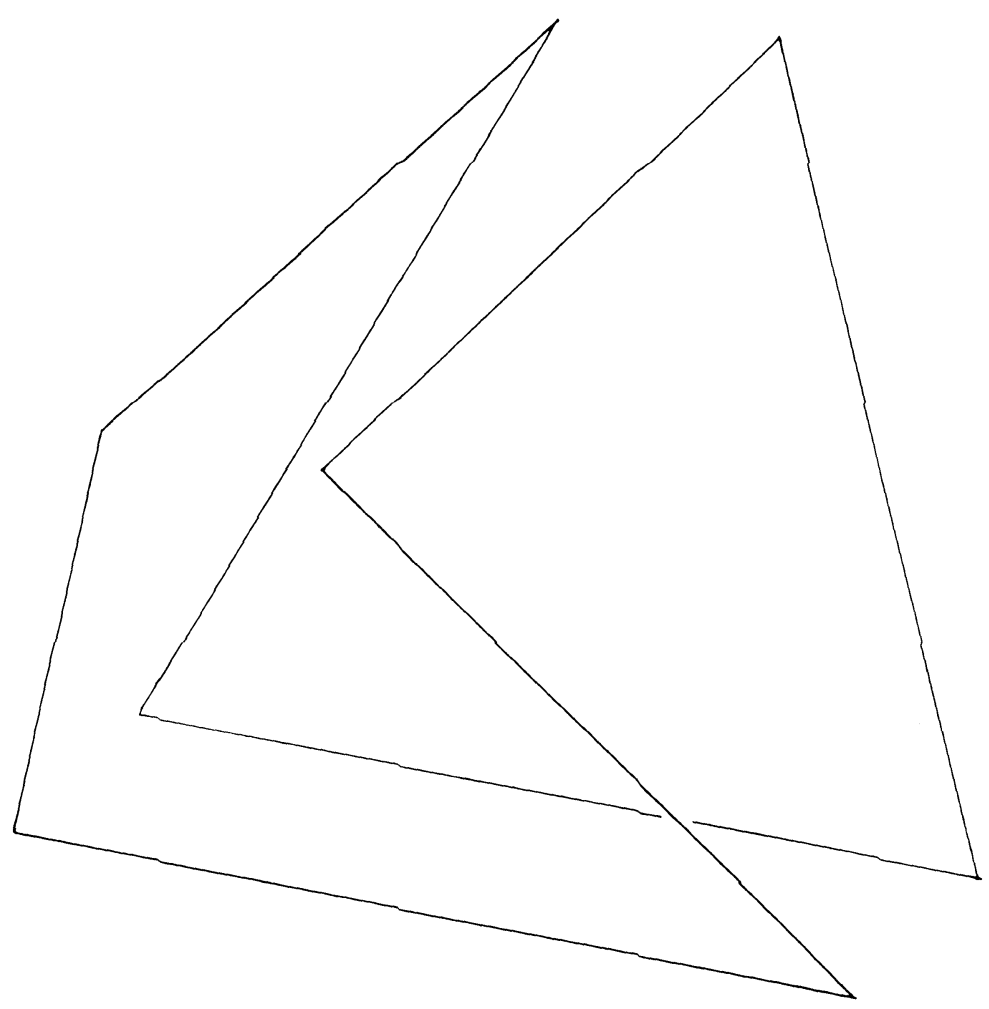

Fig. 1c. 
we had an estimate for $\Gamma$, we would also need it for the perturbed curves $\Gamma_{t}$.

This difficulty can be surmounted by defining $\Gamma_{t}$ more carefully. Let $R$ and $S$ be as above; we shall arrange that for $t$ positive, $\Gamma_{t}$ lies entirely on $R$, and for $t$ negative, $\Gamma_{t}$ lies entirely on $S$. We will then be in a position to use the theorem that an analytic curve lying on a minimizing surface bounds exactly one minimizing surface (Lemma 1 below). We then try to finish the proof as follows: Suppose for purposes of contradiction that $F_{t}$ is a minimal surface bounded by $\Gamma_{t}$. If $t$ is positive, then $F_{t}$ coincides with a portion of $R$, and if $t$ is negative, then $F_{t}$ coincides with a portion of $S$. Thus, there must be a jump discontinuity in $F_{t}$ as $t$ changes sign. We now see a second major difficulty: it has to be proved that $R$ and $S$ are distinct; that is, that $R$ is not invariant under the symmetry $\sigma$ of $\Gamma_{0}$. Happily, this has already been done, by Nitsche [6]. We now give the details of the proof.

We begin by giving the precise definition of $\Gamma_{0}$; the parametric equations for $\Gamma_{0}$ are, for $0 \leqq \theta \leqq 2 \pi$

$$
\begin{aligned}
& x=a \cos \theta-1 / 3 a^{3} \cos 3 \theta \\
& y=-a \sin \theta-1 / 3 a^{3} \sin 3 \theta \\
& z=a^{2} \cos 2 \theta
\end{aligned}
$$

Here $a$ can be taken to be 1.7 ; it has to be some number between $\sqrt{3}$ and some critical value $r_{0}$ which is about 1.68, in order that the results of [6] which we need below should apply.

LEMMA 1. Let the Jordan curve $\Gamma$ bound a minimizing surface $R$, and let $C$ be an analytic Jordan curve lying on $R$ and not entirely lying on $\Gamma$. Then $C$ bounds exactly one minimizing surface.

Proof. (cf. [4, p. 92] where a similar theorem is given). $C$ certainly bounds one minimal surface $R^{\prime}$, which is a portion of $R$. Let $S^{\prime}$ be any minimizing surface bounded by $C$; we will show $S^{\prime}$ and $R^{\prime}$ coincide. Let $S$ be the piecewise $C^{1}$ surface formed by $S^{\prime}$ together with $R-R^{\prime}$, together with $C ; S$ is bounded by $\Gamma$ and therefore has area $\geqq$ the area of $R$. Hence area $\left(S^{\prime}\right) \geqq \operatorname{area}\left(R^{\prime}\right)$. This proves that $R^{\prime}$ is a minimizing surface. Since $S^{\prime}$ is a minimizing surface, area $\left(S^{\prime}\right)=$ area $\left(R^{\prime}\right)$. Now, by [5], the surfaces $S^{\prime}$ and $R-R^{\prime}$ can be analytically extended across $C$. If they do not have a common tangent plane at each point of their common boundary, we can "round off" the corner to decrease the area, which is impossible, as $\left(R-R^{\prime}\right) \cup S^{\prime}$ has minimum area among surfaces bounded by $\Gamma$. Hence they do have a common tangent plane. But now, by analyticity, they are analytic continuations of each other. (This 
is a well-known fact about minimal surfaces which is proved by examining the associated analytic functions; see [7] for details.) But $R-R^{\prime}$ has only one analytic continuation, namely $R^{\prime}$. Hence $R^{\prime}$ and $S^{\prime}$ coincide, and the lemma is proved.

The following lemma is proved in [6], where it is used to show that $\Gamma_{0}$ bounds at least three minimal surfaces.

LEMMA 2. There is no minimizing surface bounded by $\Gamma_{0}$ which is geometrically invariant under the symmetry $\sigma$ of $\Gamma_{0}$.

We are now ready to state and prove our main theorem, that there is no local continuous dependence of a minimal surface on the boundary, for boundaries near $\Gamma_{0}$. In order to formulate such a theorem precisely, we must put a topology on the set of minimal surfaces and a topology on the space of boundary curves. In order that our (negative) theorem should be as strong as possible, we should put a strong topology on the boundary curves, and a weak topology on the surfaces. We therefore place the $C^{n}$ topology on the boundary curves, for some fixed but arbitrary $n$, and on the surfaces we use the topology induced by the Frechet distance,

$$
(F, G)=\inf _{\phi}\|F \phi-G\|
$$

where $\|\cdot\|$ is the sup norm and $\phi$ ranges over all homeomorphisms of the unit disk onto itself.

THEOREM. It is not the case that for every analytic boundary curve, a minimizing surface can be found depending locally continuously on the curve.

REMARK. The theorem is related to, but somewhat stronger than, the fact that when a minimizing surface is perturbed, it might not be minimizing any more, though still minimal. The exact definition of local continuous dependence is given above.

Proof. We have to show that if $R$ is any minimizing surface bounded by $\Gamma_{0}$, there is $\epsilon>0$ such that it is possible to perturb $\Gamma_{0}$ by an arbitrarily small amount to some $\Gamma_{t}$ which bounds no minimizing surface within $\epsilon$ of $R$. Fix some minimizing surface $R$ bounded by $\Gamma_{0}$. Let $S$ be the minimizing surface obtained from $R$ by applying the symmetry $\sigma$ of $\Gamma_{0}$, i.e., rotation by $90^{\circ}$ and reflection in the $x-y$ plane. We may also assume that $S$ has been reparametrized so that, regarding $S$ as a function from the closed unit disk $\bar{P}$ to $R^{3}$, we have $S\left(e^{i \theta}\right)=R\left(e^{i \theta}\right)$. For that matter, we may assume $R$ was so parametrized that $R\left(e^{i \theta}\right)=$ $\Gamma_{0}(\theta)$. Now, let 


$$
\Gamma_{t}(\theta)= \begin{cases}R\left((1-t) e^{i \theta}\right) & \text { if } \quad t \geqq 0 \\ S\left((1+t) e^{i \theta}\right) & \text { if } \quad t<0\end{cases}
$$

Since, by [5], $R$ and $S$ extend analytically beyond the boundary, $\Gamma_{t}$ approaches $\Gamma_{0}$ as $t$ approaches 0 . (Of course, $\Gamma_{t}$ isn't smooth as a function of $t$, but this doesn't matter.)

Here we encounter a difficulty, which for simplicity we did not mention before. Namely, why are the curves $\Gamma_{t}$ Jordan curves? In other words, why are they one-to-one? For $t$ sufficiently near zero, $\Gamma_{t}$ will be close to $R\left(e^{i \theta}\right)$ in the $C^{1}$ norm. It is not difficult to see that this would imply that $\Gamma_{t}$ is one-to-one, if we knew that the derivative of $R$ with respect to $\theta$ were never zero; that is, if we knew that $R$ has no boundary branch points. Since $R$ is a minimizing surface, this is a known result $[G-L]$. Hence, for $t$ sufficiently near zero, $\Gamma_{t}$ is a Jordan curve, and we can proceed.

By Lemma 1 , if $t \neq 0$, then there is exactly one minimizing surface bounded by $\Gamma_{t}$; it is a restriction of $R$ or $S$ to a disk of radius $1-|t|$. Take $\epsilon$ to be half the Frechet distance of $R$ and $S$. We have to check that $\epsilon>0$; but this is the content of Lemma 2. We will be finished as soon as we show that the Frechet distance $\rho\left(R_{t}, S_{t}\right)$ is at least $\epsilon / 2$ for $t$ sufficiently near 0 . This in turn follows if we show that $\rho\left(R_{t}, R\right)$ and $\rho\left(S_{t}, S\right)$ approach zero as $t$ approaches zero. This follows from the continuity of $R$ in the closed disk $-R$ can be reparametrized in the disk of radius $1-|t|$ by "shrinking"; if $t$ is small, this will not change $R(z)$ much for any $z$. This completes the proof of the Theorem.

\section{REFERENCES}

1. M. Beeson, Principles of continuous choice and continuity of functions in formal systems for constructive mathematics, (to appear).

2. - Plateau's problem and constructive mathematics, (to appear).

3. R. D. Gulliver, and F. D. Lesley, On boundary branch points of minimizing surfaces, Arch. Rational Mech. Anal., 52 (1973), 20-25.

4. H. B. Lawson, Jr., Lectures of Minimal Submanifolds, Vol. 1., Instituto de Matematica Pura e Aplicada, Conselho Nacional de Pesquisas.

5. H. Lewy, On the boundary behavior of minimal surfaces, Proc. Nat. Acad. Sci., 37 (1951), $103-110$.

6. J. C. C. Nitsche, Contours bounding at least three solutions of Plateau's problem, Arch. Rational Mech., 30 (1968), 1-11.

7. R. Osserman, A Survey of Minimal Surfaces, van Nostrand, 1969.

8. F. Tomi, A perturbation theorem for surfaces of constant mean curvature, Math Z., 141 (1975), 253-264.

Received November 4, 1975.

UNIVERSITEIT VAN AMSTERDAM

ROETERSSTRAAT 15

Amsterdam 1004, The Netherlands 




\section{Pacific Journal of Mathematics \\ Vol. 70, No. $1 \quad$ September, 1977}

William H. Barker, Noether's theorem for plane domains with hyperelliptic

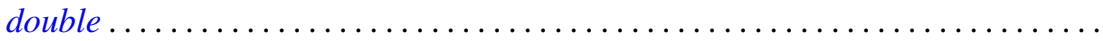

Michael James Beeson, Non-continuous dependence of surfaces of least area on the boundary curve ...................................... 11

Horst Behncke, Functions acting in weighted Orlicz algebras . . . . . . . . . . . . 19

Howard Edwin Bell, A commutativity study for periodic rings . . . . . . . . . . . 29

Peter Botta and Stephen J. Pierce, The preservers of any orthogonal group ....... 37

Douglas S. Bridges, The constructive Radon-Nikodým theorem ............. 51

James Dennis Brom, The theory of almost periodic functions in constructive

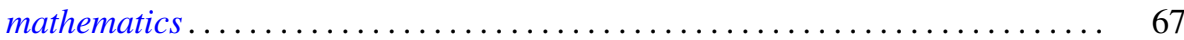

N. Burgoyne and C. Williamson, Semi-simple classes in Chevalley type groups ....

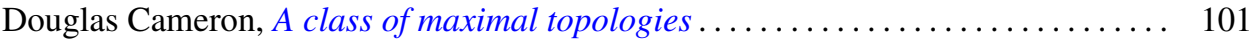

L. Carlitz, Enumeration of doubly up-down permutations . . . . . . . . . . . . . . 105

Paul Robert Chernoff, The quantum n-body problem and a theorem of

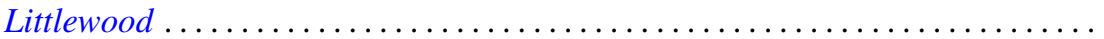

Jo-Ann Deborah Cohen, Locally bounded topologies on $F(X) \ldots \ldots \ldots \ldots \ldots \ldots$

Heinz Otto Cordes and Robert Colman McOwen, Remarks on singular elliptic

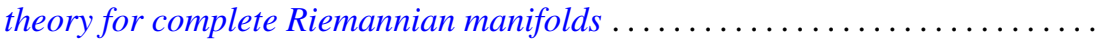

Micheal Neal Dyer, Correction to: "Rational homology and Whitehead

products"

Robert Fernholz, Factorization of Radonifying transformations

Lawrence Arthur Fialkow, A note on quasisimilarity. II ...... . .

Harvey Charles Greenwald, Lipschitz spaces of distributions on the surface of unit

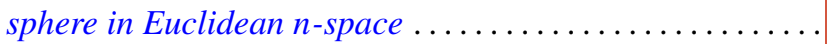

Albrecht Irle, On the measurability of conditional expectations

Tom (Roy Thomas Jr.) Jacob, Matrix transformations involving simple sequence

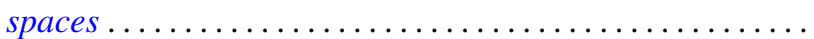

A. Katsaras, Continuous linear maps positive on increasing continuous

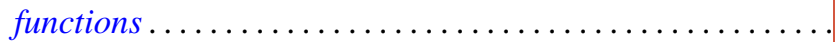

Kenneth Kunen and Judith Roitman, Attaining the spread at cardinals of cofinality

Lawrence Louis Larmore and Robert David Rigdon, Enumerating normal bundles

of immersions and embeddings of projective spaces ...... . .

Ch. G. Philos and V. A. Staïkos, Asymptotic properties of nonoscillatory solutions of differential equations with deviating argument .

Peter Michael Rosenthal and Ahmed Ramzy Sourour, On operator algebras containing cyclic Boolean algebras...

Polychronis Strantzalos, Strikt fast gleichgradig-stetige und eigentliche

Aktionen ...

Glenn Francis Webb, Exponential representation of solutions to an abstract

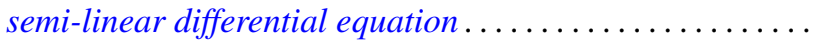

